Download

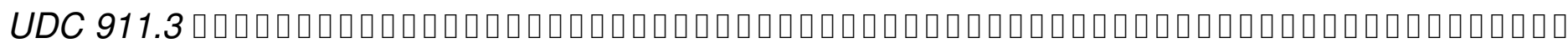

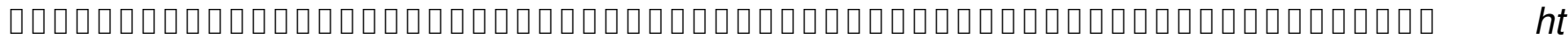
tps

$: / /$

doi

org

$/ 10.17721 / 2308-135$

$X$ .2020 .55 .

3 12

Shushniak Volodymyr Mykolayovych, PhD, Associate Professor, Ivan Franko National University of Lviv. Lviv, Ukraine, e-mail:

shushniak

@

gmail

com

Savka Halyna Stepanivna, Ivan Franko National University of Lviv. Lviv, Ukraine, e-mail: savka.halyna@gmail.com 
Shandra Yuri Yaroslavovych, Ivan Franko National University of Lviv. Lviv, Ukraine, e-ma il: riyshandra

@ gmail com

\section{THE MAIN EUROPEAN WATERSHED OF UKRAINE AND ITS IMPORTANCE IN TERMS OF TOURISM}

Objective: On the basis of general geographical analysis to reveal the tourist potential of the Main European watershed on the territory of Ukraine.

Method. General scientific methods of analysis, synthesis and generalization as well as cartographic methods by the means of GIS have been used.

Results:The peculiarities of the geographical location of the Main European Watershed on a European scale have been analyzed. New facts from the history of its research have been presented. A hypsometric profile along the watershed line has been constructed, its morphometric parameters have been determined.

With the help of detailed cartographic analysis, the Ukrainian section of the Main European Watershed line has been identified. Its location in relation to the physical and geographical zoning has been determined. A regional analysis of tourist facilities attractiveness on the watershed line has been conducted. Cross-border and domestic transport opportunities for 
access to the watershed line have been assessed. The coordinate reference of the most important orohydrographic elements - the points of watersheds intersection of large transboundary rivers basins: Danube, Vistula, Dniester, Dnieper, as well as their tourist significance have been specified. Possibilities of tourist use of nature reserve fund objects which are located on the watershed line have been revealed, in particular of such national natural parks, as Uzhanian National Nature Park, Boikivschyna National Nature Park, Yavorivs kyi National Park

Northern Podillia, Shatsk National Natural Park. The importance of the watershed for sustainable (ecologically oriented) tourism development in international biosphere reserves: "The Eastern Carpathians" (Slovak-Ukrainian-Polish), "Roztochia" (Ukrainian-Polish), "Western Polissya" (Polish-Ukrainian-Belarusian) has been demonstrated. Touristic assessment of historical and cultural sites located on the watershed line has been conducted. The importance of the city of Lviv as a separate tourist destination has been emphasized. Environmental problems related to the development of tourist resources have been outlined. Selected tourist clusters of the destination have been identified, the possibilities of their use have been revealed. The Main European Watershed specifics in general tourist resources classification have been emphasized.

Scientific novelty: For the first time, the Main European Watershed has been considered as a separate integrated tourist destination, its detailed geographical analysis has been performed, and a cluster approach has been used to assess the tourist resources located on the watershed line.

Practical significance. The results of the study will contribute to the integration process of Ukraine into the European tourist area, give impetus to new tourist routes planning, promote the development of tourist resources of national natural and regional landscape parks, support tourist clusters planning and developing.

Key words: Main European watershed, tourist destination, tourist cluster, river basin.

\section{References}

1. Apollov B. A. Ucheniye o rekakh / Apollov B. A. - M.: Izdatelstvo Moskovskogo universiteta, 1951. - $522 \mathrm{~s} . \quad$ (In Russian). 2. Anufriyev V. N. Upravleniye transgranichnym basseynom Dnepra: subbasseyn reki Pripyati: [monografiya] / V. N. Anufriyev, K. A. Aliyev, S. A. Afanasyev, Ye. P. Bogodyazh, V. N. Bosak; red.: A. G. Obodovskiy, A. P. Stankevich, S. A. Afanasyev. - K.: Kafedra, 2012. - 447 c. 
(

In

Russian

).

3.

Herenchuk

$\mathrm{K}$

Do

chetvertynnoyi

paleoheohrafiyi

Sans

(

ko

Dnistrovs

口

koho

mezhyrichchya

/I

Paleoheohrafichni

umovy

terytoriyi

Ukrayiny

$\mathrm{v}$

pliotseni

i

antropoheni

/

$\mathrm{K}$

Herenchuk

M

S

Demedyuk

M 
V

Zdenyuk

-

$\mathrm{K}$

$\therefore$

Nauk

dumka

, 1966. -

$\mathrm{S}$

$.5-19 .($

In

Ukrainian

).

4.

Heohrafichna entsyklopediya Ukrayiny: v 3 t. T. 1 / red. kol.: O. M. Marynych ta in. - 1989. $416 \mathrm{~s}$.

(

In

Ukrainian

).

5.

Derzhavnyy

vodnyy

kadastr

oblik

poverkhnevykh

vodnykh

obektiv

[

Elektronnyy

resurs

]. -

Rezhym

dostupu

http://geoportal.davr.gov.ua

$: 81 /$ (

In 
v551

Written by Administrator

Wednesday, 10 February 2021 15:51 - Last Updated Thursday, 14 July 2022 16:22

Ukrainian

) .

6.

$$
\begin{aligned}
& \text { Zahalna } \\
& \text { hidrolohiya } \\
& \text { : [ } \\
& \text { pidruchnyk } \\
& \text { ]/ } \\
& \text { V } \\
& \text { K }
\end{aligned}
$$

Khilchevskyy

$\mathrm{O}$

$\mathrm{H}$

Obodovskyy

i

V

Hrebin

ta

in

. -

$\mathrm{K}$

$\therefore$

Vydavnycho

polihrafichnyy

tsentr

«

Kyyivskyy

universytet

», 2008. - 399

$\mathrm{S}$

. (

In

Ukrainian

). 
7.

Zaleskyy

I

I

Shatske

poozerya

:

Heolohichna

budova

ta

hidroheolohichni

umovy

I

I

I

Zaleskyy

F

V

Zuzuk

V

$\mathrm{H}$

Melnychuk

V

V

Mateyuk

H

i

Brovko 
UNSPECIFIED. Skhidnoyevrop. nats. un-t im. Lesi Ukrayinky, Lutsk. - T. 1, 2014. - 190 s. (

In

Ukrainian

).

8.

Karta Polesya s oboznacheniyem liniy nivelirovki [Yelektronniy resurs]. - Rezhim dostupu: http://www.etomesto.ru/map-belarus polesie hodovye-linii-1874/

In

Russian

).

9.

Kruber A. A. Obshcheye zemlevedeniye. M.-L.: Uchpedgiz,1938. - 469 s.

(

In

Russian

).

10.

Lviv: Kompleksnyy atlas / vidp. red. O. I. Shabliy. - K.: DNVP «Kartohrafiya», 2012. - 192 s. ( In

Ukrainian

).

11.

Pryroda Lvivskoyi oblasti / za red. K. I. Herenchuka. - Lviv: Vyshcha shkola, 1972. - 151 s. In

Ukrainian

)

12.

Pryroda Volynskoyi oblasti / za red. K. I. Herenchuka. - Lviv: Vyshcha shkola, 1975. - 147 s. (

In

Ukrainian 
)

13.

Rudnytskyy S. Znadoby do morfolohiyi Pidkarpatskoho stochyshcha Dnistra / S. Rudnytskyy / Zb. matem.-pryrod. sektsiyi NTSH. - T. 2. - 1907. - 79 s.

(

In

Ukrainian

)

14.

Russkoye geograficheskoye obshchestvo [Yelektronniy resurs]. - Rezhim dostupu:

https://www.rgo.ru/ru/grant/landshaftno-istoricheskaya-ekspediciya-ural-granica-evropy-i-azii In

Russian

).

15.

Savka

$\mathrm{H}$

Shushnyak

$\mathrm{V}$

Landshaftno

sozolohichnyy

analiz

heosaytiv

Ukrayinskoho

Roztochya

I

Heoturyzm

:

praktyka

i

dosvid

Mater. II mizhnar. nauk.-prakt. konf. (5-7 travnya 2016 r., Lviv). - Lviv: NVF «Karty i Atlasy», 2016. - S. 64-66. 
(

In

Ukrainian

)

16.

Savka H. S., Shushnyak V. M. Landshaftno-krayeznavchi marshruty v okolytsyakh Roztots $\square$ koho landshaftnoho-heofizychnoho statsionaru / Dovhoterminovi sposterezhennya dovkillya: dosvid, problemy, perspektyvy: materialy Mizhnar. nauk. seminaru (Lviv-Bryukhovychi, 10-12 travnya 2019 r.). - Lviv: LNU imeni Ivana Franka, 2019. - S. 176178.

(

In

Ukrainian

)

17.

Stepaniv O. Suchasnyy Lviv: [putivnyk] / Olena Stepaniv. - Krakiv; Lviv, 1943. - 168 s.

(

In

Ukrainian

)

18.

Osnovy zahalnoyi hidrolohiyi / za red. Levkivskoho S.S. - K: Vyshcha shkola, 1975. - 192 s.

(

In

Ukrainian

)

19.

Shushnyak V., Savka H. Problemy fizyko-heohrafichnoyi rehionalizatsiyi Roztochchya // Fizychna heohr. ta heomorf. - K.: VHL Obriyi, 2014. - Vyp. 46, T. 2. - S. 222-230).

In

Ukrainian

) 
20.

Shushnyak V. M., Savka H. S., Verheles YU. Rezultaty inventaryzatsiyi vodnykh obektiv m. Lvova / Visnyk Lvivskoho universytetu. Seriya: Heohrafichna / Lvivskyy nats. un-t im. I. Franka. - Lviv, 2014. - Vyp. 48. - S. 322-327.

(

In

Ukrainian

)

21.

Shushnyak V., Shelest D., Savka H., Shuliha YE. Osoblyvosti fizyko-heohrafichnoho polozhennya Natsional noho pryrodnoho parku «Pivnichne Podillya» // Pryroda Volyni y Podillya: doslidzhennya ta okhorona: Materialy Pershoyi mizhnarodnoyi naukovo-praktychnoyi konferentsiyi (m. Brody, 3-5 chervnya 2015 roku). - Lviv: Liha-Pres, 2015. - S. 63-68. (

In

Ukrainian

)

22.

Yavorskyy B. I., Savka H. S., Shushnyak V. M. Novi heoturystychni marshruty vizyt-tsentru "Vereshchytsya» Yavorivskoho natsionalnoho pryrodnoho parku / Pryrodookhoronni, ekoosvitni, rekreatsiyno-turystychni ta istoryko-kulturni aspekty staloho rozvytku Roztochchya: materialy Mizhnar. nauk.-prakt. konferentsiyi, prysvyachenoyi 20-richchyu stvorennya Yavorivskoho natsionalnoho pryrodnoho parku. - smt Ivano-Frankove; Lviv: ZUKTS, 2018. - S. 299-308.

(

In

Ukrainian

)

23.

Zlewnie rzek Bugu i Narwi. Zasoby wodne i przyrodnicze: Monografia, Wyższa Szkoła Ekologii i Zarz aczania w Warszawie. - Warszawa, 2007. - 448 s.

(In Polish).

24.

J

a 
$\mathrm{hn}$

A

P

ół

nocna

kraw

e

d

ź

Podola

i

historia

jej

bada

ń // C

zasopismo

geograficzne

/I

Polskie

towarystwo

geograficzne

$\bar{T}$

LX

zeszyt

3.

1989.

$\mathrm{S}$

.353

-

365.

(In Polish).

25.

Plan

$\mathrm{kr}$

ó

lewskiego

sto

t 
v551

Written by Administrator

Wednesday, 10 February 2021 15:51 - Last Updated Thursday, 14 July 2022 16:22

\section{ecznego}

miasta

Lw

ó

wa

(

z

entklaw

ą

Jalowiec

takze

Masz

ó

w

I cześć.

Lwów.

$-$

1890.

(In Polish).

26.

Pol

W

ół

nocny

wsch

ó

d

Europy

pod

e

dem

natury

Prelekcje

W

\section{Pola}

Z

. 1 ,

Drukarnia

Uniwersytecka 


$$
\begin{aligned}
& \text { Krak } \\
& \text { ó }
\end{aligned}
$$

$$
, 1851 .
$$

$-$

S

.94

111.

(In Polish).

27.

Rudnicki

S

Polemika w sprawie historii doliny Dniestru // Kosmos - Lwów, 1907. - Rocz. 32. - S. 91

92. (In Polish).

28.

Romer E. Odpowiedź Dr. Stefanowi Rudnickiemu w sprawie historyi doliny Dniestru. 1907

s. 93-94

Kosmos. -

Lwów, 1907. -

Rocz. 32. (In Polish).

29.

Roth

M

.,

$\mathrm{R}$

Nobis

V

Stetsiuk

Kruhlov

( 


\section{Eds}

) (2008):

Transformation

processes

in

the

Western

Ukraine

\section{Concepts}

for

a

sustainable

land

use

Wei

B

ensee

Verlag

Berlin

2008. 602

pages

30.

$\mathrm{S}$

t

ownik

geograficzny

$\mathrm{Kr}$

ó

lestwa

Polskiego

i

innych

kraj

ó

w

S

t

owia

ń

skich

T 
.5: [

Kutowa

Wola

$-$

Malczyce

]/

Red

$\therefore$

Chlebowski

Bronis

$t$

aw

Sulimierski

Filip

Walewski

W

t

adys

t

aw

.-

Warszawa

, 1884. - 960

$\mathrm{S}$

(

In

Polish

).

31.

WISE

Water Information System for Europe is the European information gateway to water issues [Yelektronniy resurs]. - Rezhim dostupu:

http://water\&gt;

$\therefore$

europa

$\underline{\mathrm{eu} /}$

32.

2rouesenliberté [Yelektronniy resurs]. - Rezhim dostupu: https

$: / /$ 
v551

Written by Administrator

Wednesday, 10 February 2021 15:51 - Last Updated Thursday, 14 July 2022 16:22

WWW

.2

rouesenliberte

$\mathrm{fr}$

1

divisiora

-2012/

(In

French)

Надійшла до редколегії /Received Editorial Board 17.06.2020 